\title{
Evaluation of the Safety and Brain-Related Tissues Distribution Characteristics of TAT-HaFGF via Intranasal Administration
}

\author{
Jinmei Xu, ${ }^{b, \#}$ Qi Xiang, ${ }^{a, c, \#}$ Junhui Su, ${ }^{b}$ Penghui Yang, ${ }^{a}$ Qihao Zhang, ${ }^{a}$ Zhijian Su, ${ }^{a, c}$ \\ Fei Xiao, ${ }^{d}$ and Yadong Huang ${ }^{*, a, c}$ \\ ${ }^{a}$ Institute of Biomedicine, Jinan University; ${ }^{b}$ College of Pharmacy, Jinan University; ${ }^{c}$ National Engineering Research \\ Center of Genetic Medicine, Jinan University; and ${ }^{d}$ Department of Pharmacology, School of Medicine, Jinan \\ University; Guangzhou 510632, China.
}

Received January 9, 2014; accepted April 16, 2014

\begin{abstract}
Disabilities triggered by neurodegeneration mainly result in mortality in the elderly, and patients with neurodegenerative disease also display deficits in olfactory function. Therefore drug distribution to the brain through intranasal administration has become one of the most difficult challenges in the treatment of central nervous system (CNS) diseases. TAT-human acidic fibroblast growth factor (HaFGF) is a new fused protein retaining the neuroprotective activities of $\mathrm{HaFGF}$, and is a promising prospect in the treatment of neurodegenerative diseases. TAT (a cell-penetrating peptide) contains a high relative abundance of positively charged amino acids such as lysine and arginine, which have a powerful attraction to the negatively charge on the nasal epithelial membrane. The present study focused on the evaluation of the safety and absorption characteristics of TAT-HaFGF following intranasal administration. After TAT-HaFGF intranasal administration $(100,300,600 \mu \mathrm{g} / \mathrm{kg})$ for 5 weeks, hematoxylin-eosin (HE) staining showed no pathology in any of the investigated tissues and organs. The expression of olfactory marker protein (OMP) was observed with immunohistochemical staining, which showed no altered expression in the sensory neurons of the nasal epithelium. Nasal ciliotoxicity studies carried out using an in situ palate model and optical microscope showed that TATHaFGF had no nasal ciliotoxicity. The distribution of the TAT-HaFGF following intranasal administration was assessed using a radioisotopic tracing method. Radioactivity was observed in the brain after $15 \mathrm{~min}$. This became stronger at $30 \mathrm{~min}$ and weaker at $1 \mathrm{~h}$. All of the results confirmed the in vivo safety of TAT-HaFGF via intranasal administration.
\end{abstract}

Key words TAT-human acidic fibroblast; intranasal administration; mucosal adsorption; safety evaluation

Neurodegenerative disease occurs in specific regions of the brain, and is classically associated with memory problems, olfactory deficits and movement disorders. ${ }^{1-3)}$ Intranasal drug administration has been shown to increase the delivery of peptides of a particular size and charge to the central nervous system (CNS) for the treatment of CNS diseases. ${ }^{4,5)}$ For example, intranasal administration of the $26.5 \mathrm{kDa}$ recombinant human nerve growth factor in rats resulted in CNS concentrations 10-45-fold higher (depending on the brain region) than concentrations observed following intravenous administration. ${ }^{6}$ Similarly, intranasal delivery of hypocretin-1 increased targeting to various brain regions by $7-13$-fold. ${ }^{7)}$ Enhancement of delivery and targeting to the brain has been demonstrated with the intranasal delivery of a wide range of compounds, including galanin-like peptide, ${ }^{8)}$ insulin-like growth factor, ${ }^{9)}$ insulin, ${ }^{10)}$ interferon $\beta-1 \mathrm{~b},{ }^{11)}$ transforming growth factor- $\beta 1,{ }^{12)}$ and hypocretin-1. ${ }^{7}$

Human acidic fibroblast growth factor (HaFGF) is involved in the regulation of synaptic plasticity and learning and memory processes through the improvement of cholinergic nerve functions. Furthermore, recombinant polypeptide fibroblast growth factor has been applied in clinical settings for the treatment of cardiovascular and neurodegenerative diseases. ${ }^{13)}$ Delivery of bioactive peptides across the blood-brain barrier (BBB) is generally restricted. Recent research showed that a 9-amino acid motif from, the protein transduction domain of human immunodeficiency virus-1 (HIV) Tat, has a power-

The authors declare no conflict of interest.

\# These authors contributed equally to this work. ful attraction to the negatively charge on the nasal epithelial membrane. ${ }^{14)}$ This feature was attributed to a 9-amino acid stretch of basic amino acids, with the sequence Arg-Lys-LysArg-Arg-Gln-Arg-Arg-Arg. ${ }^{15-17)}$ When partially denatured, the chimeric protein is capable of crossing the BBB of adult mice. $^{18)}$ Our previous study has shown that modified TATHaFGF could penetrate the BBB when administered intravenously, ${ }^{19)}$ however, this method of injection is not specifically targeted to the brain. With intranasal administration, drugs can directly reach the CNS for the treatment of CNS diseases via nose-to-brain pathways, minimizing systemic exposure and decreasing side effects. ${ }^{20}$ In our previous work, intranasal administration of TAT-HaFGF was found to significantly increase the concentration of HaFGF in the brain. Importantly, TAT-HaFGF enhanced the learning and memory abilities of senescence-accelerated mouse prone-8 (SAMP8) mice (a mouse model of Alzheimer's disease), as demonstrated by the Morris water maze test, and promoted the function of the cholinergic system shown by the measurement of acetylcholine (Ach), acetylcholinesterase (AChE) and choline acetyltransferase (ChAT). TAT-HaFGF also significantly reduced amyloidbeta $(\mathrm{A} \beta)_{1-42}$ deposits as well as the levels of soluble $\mathrm{A} \beta$ forms in the mice brains and prevented apoptosis in the neurons. ${ }^{21)}$ However it is unclear whether repeated delivery of fluid into the nares will increase the risk of damage to the nasal cavity and olfactory bulbs.

The present study focused on the safety evaluation of repeated delivery of fluid into the nares and the absorption characteristics of TAT-HaFGF following nasal administration. 


\section{MATERIALS AND METHODS}

Animals Eight male and 8 female bufo bufo gargarizans weighing 60-80 g and 20 male and 20 female Sprague-Dawley (SD) rats weighting 200-220g were purchased from the Medical Experimental Animal Center of Guangdong Province (Guangzhou, China).

All rats were kept under conditions of controlled temperature $\left(24 \pm 1^{\circ} \mathrm{C}\right)$, humidity $(50-60 \%)$ and a light/dark cycle of $12 / 12 \mathrm{~h}$. Animals were allowed free access to a standard rodent diet and water. All experiments were conducted according to the Chinese Guidelines for Animal Care and Use, and were approved by the Animal Ethics Committee of the Chinese Academy of Medical Science.

Materials TAT-HaFGF recombinant fusion proteins were expressed and purified in our own lab by using the previously described method, ${ }^{22)}$ stored at $-80^{\circ} \mathrm{C}$ and were dissolved in $0.9 \%$ normal saline to create an experimental dosage solution for use in drug administration. Ephedrine hydrochloride was obtained from the National Institute for the Control of Pharmaceutical and Biological Products. Deoxysodium cholate was provided by Sinopharm Chemical Reagent Corporation Limited. An SABC immunohistochemical kit was purchased from Boster Biotech. A Rabbit monoclonal anti-olfactory marker protein (OMP) antibody was purchased from Abcam. Anti-TAT was purchased from Cell Application. A kit for the ${ }^{125}$ I labeling of proteins was provided by PerkinElmer, Inc. ${ }^{\mathrm{TM}}$

Safety Evaluation of TAT-HaFGF by Intranasal Administration. Nasal Ciliotoxicity Nasal ciliotoxicity studies were carried out using an in situ palate model. ${ }^{18)}$ In brief, after pithing bufo bufo gargarizans bull toads, their palates were excised and blood on the palate was cleaned. The isolated palates were placed on a glass slide and treated with $0.5 \mathrm{~mL}$ of normal saline, TAT-HaFGF $(1.4 \mathrm{mg} / \mathrm{mL})$, ephedrine hydrochloride $(10 \mathrm{mg} / \mathrm{mL})$ and deoxysodium cholate $(10 \mathrm{mg} / \mathrm{mL})$. To evaluate the influence of TAT-HaFGF drops on the movement of toad palate mucosa cilia, we continuously observed the mucosa for $12 \mathrm{~h}$ and recorded the time span of continuing segmental motion of the toad palate cilia after sufficient effect by drugs under an optical microscope (IX71, Olympus). ${ }^{23)}$

Effects of Repeated Intranasal Administration of TATHaFGF on SD Rats Animal Grouping and Drug Treatment: Rats were anesthetized with intraperitoneal (i.p.) pentobarbital sodium $(40 \mathrm{mg} / \mathrm{kg})$ and were placed in a supine position and a pad was inserted under the dorsal neck to extend the head back toward the supporting surface. An average of $50 \mu \mathrm{L}$ of total TAT-HaFGF $(100,300,600 \mu \mathrm{g} / \mathrm{kg})$ and $0.9 \%$ saline was then administered by pipette in $5 \mu \mathrm{L}$ drops, alternating between each naris every $2 \mathrm{~min}$, over a total of $15 \mathrm{~min} .^{24)}$ Drugs were delivered intranasally to rats every $2 \mathrm{~d}$ for 5 weeks. The details of animal groups and drug treatments are shown in Table 1 .

Table 1. Animal Grouping and Drug Treatment

\begin{tabular}{lccc}
\hline \hline Group & Treatment & Dosage $(\mu \mathrm{g} / \mathrm{kg})$ & Number of rat \\
\hline Control & Saline & - & 5 \\
T100 & TAT-HaFGF & 100 & 5 \\
T300 & TAT-HaFGF & 300 & 5 \\
T600 & TAT-HaFGF & 600 & 5 \\
\hline
\end{tabular}

Sample Processing: After 5 weeks, rats were fasted overnight after the last delivery, then anesthetized and flushed via cardiac perfusion with saline. The heart, liver, spleen, lung, kidney, olfactory bulbs, and noses of the animals were collected. The main organs were immersion-fixed in 4\% paraformaldehyde for $24 \mathrm{~h}$. The noses were immersion fixed and decalcified in $20 \%$ formic acid and formaldehyde decalcified fluid for $12 \mathrm{~h}$, and then placed in a new decalcified fluid for $12 \mathrm{~h}$ to ensure complete decalcified inside the nares. Tissue was paraffin embedded, and $3 \mathrm{~mm}$ horizontal sections were cut using a paraffin slicing machine and stored at $4{ }^{\circ} \mathrm{C}$ for later use. Then the slides prepared for immunohistochemistry and HE staining.

OMP Immunohistochemical Staining: Olfactory marker protein $(\mathrm{OMP})$ is a $19 \mathrm{kDa}$ intracellular protein expressed at high levels in OSNs in many vertebrate species, with very limited expression elsewhere in the nervous system. The molecular genetics and structure of OMP are now well understood. OMP is expressed late in cellular development and is widely used as a marker of mature OSNs, which can reflect the integrity of olfactory sensory neurons. ${ }^{25)}$ Immunohistochemical staining of OMP was performed to detect the distribution of olfactory nerves in the nasal mucosa and olfactory bulbs of mice. Briefly, paraffin-embedded brain sections were stained according to the protocols of the SABC immunohistochemical kit (Boster Biotech, China). Paraffin sections were dewaxed, rehydrated, and treated in a $3 \% \mathrm{H}_{2} \mathrm{O}_{2}$ solution for $20 \mathrm{~min}$ in order to eliminate endogenous peroxidase activity. Then, sections were placed in a $70 \%$ formic acid solution for $20 \mathrm{~min}$ for antigen retrieval. After being rinsed in phosphate buffered saline (PBS), sections were incubated in blocking solution ( $5 \%$ bovine serum albumin) for $1 \mathrm{~h}$ at $37^{\circ} \mathrm{C}$. They were then incubated overnight with rabbit OMP monoclonal antibody (1:500, Abcam, U.S.A.) in a humidified chamber. The sections were incubated with the secondary antibody, horseradish peroxidase-conjugated-goat anti-rabbit immunoglobulin $\mathrm{G}$ (IgG) $(1: 200)$, for $1 \mathrm{~h}$ at $37^{\circ} \mathrm{C}$. After 3,3-diaminobenzidine (DAB) and hematoxylin staining, the sections were dehydrated, cleared in xylene, and covered with neutral balsam. Sections from all experimental groups were photographed through a light microscope (IX71, Olympus), and micrographs of five sections per group were analyzed using Image-Pro plus 6.0 software to measure the number of OMP positive plaques in the nasal mucosa and olfactory bulb. The percentage of the total area of epithelium and lamina propria of the nasal mucosa or olfactory glomerular layer of the olfactory bulb in the micrograph that contained OMP deposit areas was also semiquantitatively analyzed by the above software.

Mucosal Absorption Characteristics of Intranasal Administration TAT-HaFGF Radiolabeling and Purification: The distribution of TAT-haFGF following intranasal administration was assessed using a radioisotopic tracing method. TAT-HaFGF was radiolabelled using a $\mathrm{Na}^{125} \mathrm{I}$ iodogen method described previously. ${ }^{26)}$ Briefly, $1 \mathrm{mCi}$ of $\mathrm{Na}^{125} \mathrm{I}$ was added into an iodogen-coated tube with $1 \mathrm{~mL}$ of $1.47 \mathrm{mg} / \mathrm{mL}$ TAT-HaFGF to react for $5 \mathrm{~min}$ at $27-29^{\circ} \mathrm{C}$. The radiolabelled TAT-HaFGF was collected using Sephacryl S-100 HR (Pharmacia). The radioactivity was measured with a $\gamma$-counter (WALLAC 1470 WIZARD $^{\circledR}$ Gamma Counter, PerkinElmer, Inc.).

Intranasal Administration of ${ }^{125}$ I-TAT-HaFGF: An average of $50 \mu \mathrm{L}$ of total ${ }^{125} \mathrm{I}$ labeled TAT-HaFGF $(300 \mu \mathrm{g}$ TAT- 
$\mathrm{HaFGF} / \mathrm{kg}$ body weight; ratio of unlabeled TAT-HaFGF to ${ }^{125}$ I-TAT-HaFGF, $3: 1$ ) and $0.9 \%$ saline was then administered according to the above methods of administration.

Sampling and Measurement of ${ }^{125}$ I-TAT-HaFGF Content in Tissues: Rats were administered ${ }^{125}$ I-TAT-HaFGF intranasally, but allowed to recover from initial pentobarbital anesthesia and then reanesthetized prior to final blood sampling and transcardial perfusion $(15,30,60 \mathrm{~min}$ following the start of administration). After gross dissection of the thymus, olfactory bulb, brainstem, hypophysis, cerebellum, spinal cord, and nasal cavity, each sample was placed in a $5 \mathrm{~mL}$ tube and the wet weight was determined with a microbalance prior to gamma counting. Tissue concentrations were calculated using a $\gamma$ counter (WALLAC 1470 WIZARD Gamma Counter, PerkinElmer, Inc.).

Transport Visualization of TAT-HaFGF: Rats were administered $50 \mu \mathrm{L}$ of TAT-HaFGF $(300 \mu \mathrm{g}$ TAT-HaFGF $/ \mathrm{kg}$ body weight) intranasally for once using the previously described method. Thirty minutes after intranasal administration of TAT-HaFGF, rats were anesthetized and perfused with saline, the olfactory bulbs and noses were dissected out and prepared for immunofluorescence. The remainder of the nose was immersion fixed and decalcified in $20 \%$ formic acid and formaldehyde decalcified fluid for $24 \mathrm{~h}$. Tissue was embedded with O.C.T compound (McCormick, U.S.A.), and $10 \mathrm{~mm}$ horizontal sections were cut with a cryomicrotome (Leica, Germany) and stored at $-20^{\circ} \mathrm{C}$ for later use. Sections were immersed in a PBS solution for $10 \mathrm{~min}$ in order to eliminate O.C.T compound. Then, sections were placed in a $0.2 \%$ trition solution for $10 \mathrm{~min}$. After being rinsed in PBS, sections were incubated in $5 \%$ BSA solution for $1 \mathrm{~h}$ at $37^{\circ} \mathrm{C}$. They were then incubated overnight with rabbit OMP monoclonal antibody (1:500, Abcam, U.S.A.) and anti-TAT antibody (1:500, Cell Application, U.S.A.) in a humidified chamber. The sections were incubated with the secondary antibody, DyLight ${ }^{\circledR}$ 488goat anti-rabbit IgG $\left(1: 200\right.$, Abcam, U.S.A.) and DyLight ${ }^{\circledR}$ 594 -donkey anti-mouse IgG (1:200, Abcam, U.S.A.), for $1 \mathrm{~h}$ at $37^{\circ} \mathrm{C}$. Confocal microscopy (LSM 700, Germany) was used to localize the TAT-HaFGF (anti-TAT, Cell Application).

Statistical Analysis All data are expressed as mean \pm S.E.M. Statistical differences were determined using one-way ANOVA, followed by post hoc analysis of Dunnett for multigroup comparison and $p<0.05$ was considered statistically significant.

\section{RESULTS}

Safety Evaluation of Intranasal Administration. Nasal Ciliotoxicity Deoxysodium cholate, used here as a positive control, inhibits cilial movement when acting on toad mucosa cilia for ten minutes. Ephedrine hydrochloride cannot weaken the continuing movement of toad mucosa cilia in vitro so was therefore used as a negative control. Optical microscopic observation of toad palates showed that there were a great number of cilia with a fast rate of beating on the edge of mucosa treated with TAT-HaFGF for $420 \mathrm{~min}$ (Fig. 1), and that the duration of ciliary movement lasted for $638 \mathrm{~min}$, which is comparable with those treated with ephedrine hydrochloride (650 min) and far longer than those treated with the deoxysodium cholate (only $10 \mathrm{~min}$ ) (Table 2). The results suggest that
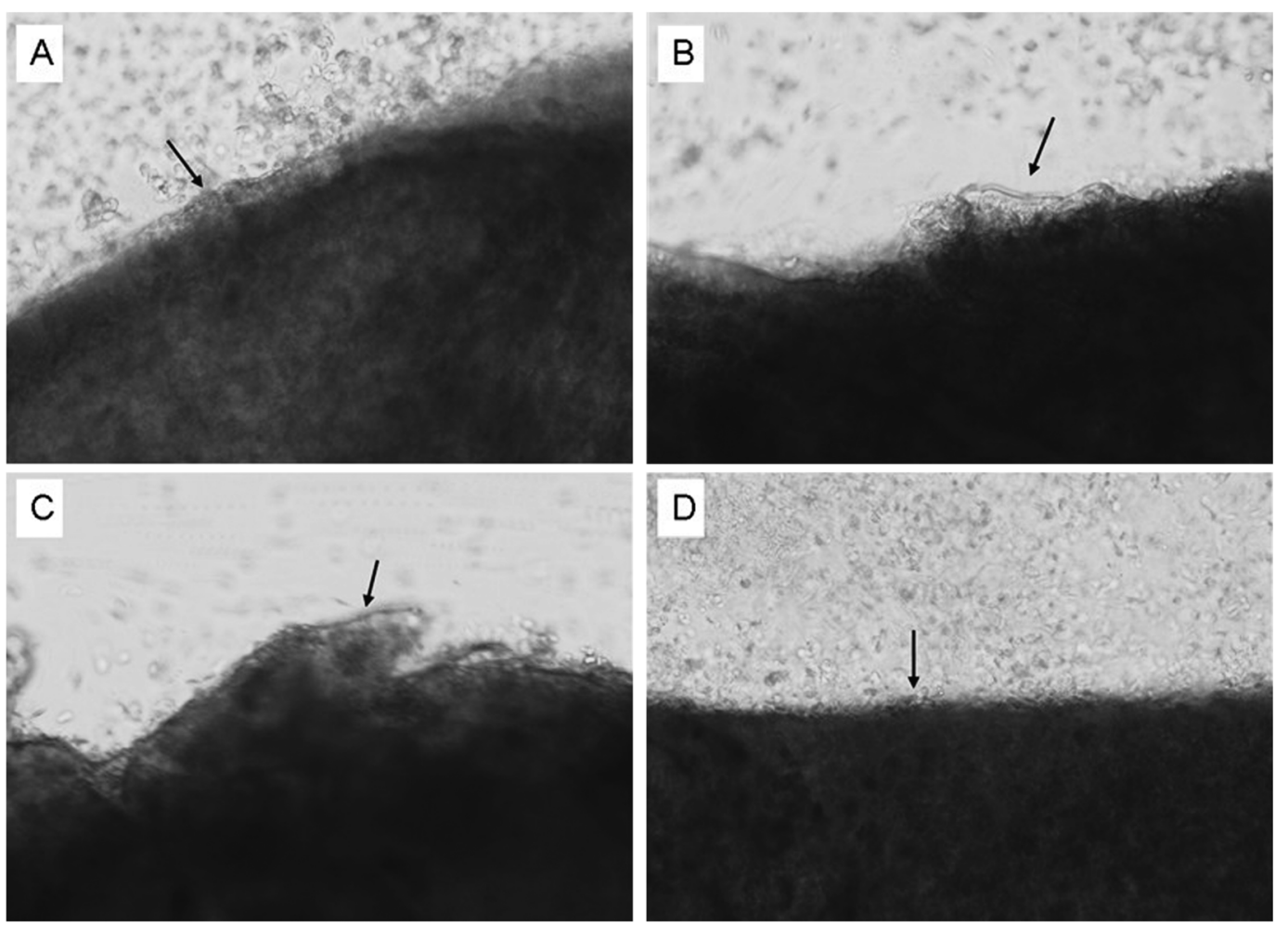

Fig. 1. Optical Microscopic Images of (A) Saline, (B) Negative Control (Ephedrine Hydrochloride, Cilia on the Mucosa Intact $7 \mathrm{~h}$ after Treatment), (C) TAT-HaFGF (Similar Phenomenon with That of Saline Visualized $7 \mathrm{~h}$ after Treatment), (D) Positive Control (Deoxysodium Cholate Solution, no Cilia on the Mucosa Observed but a Few Exfoliated Cilia 10 min after Treatment)

Cilia are indicated by arrows $(40 \times, n=4$ for each group). 
TAT-HaFGF drops caused no remarkable injury to the function of toad mucosa cilia.

Effect of TAT-HaFGF on the Nasal Cavity and Olfactory Bulbs of SD Rats Since intranasal administration results in a high concentration of TAT-HaFGF in the nose and olfactory bulbs, damage to the integrity of the nasal cavity and olfactory bulbs and the number of olfactory sensory neurons is possible. To address this problem, noses and olfactory bulbs from intranasal treated animals were processed as described in Materials and Methods. Sections were then visualized with a light microscope to ascertain the morphology of the nasal epithelium. Figures $2 \mathrm{a}$ and $3 \mathrm{a}$ demonstrate that there were no gross morphological changes or outward damage visible in any of the groups examined. Images were processed by Image-pro plus. The numbers of OMP-positive cells in the epithelium and lamina propria of the nasal mucosa or olfac-

Table 2. The Time Span of Toad Palate Cilium Continuing Segmental Motion

\begin{tabular}{lcc}
\hline \hline Group & Time of swing/min & Relative to normal saline \\
\hline Normal saline & $655 \pm 22$ & $100 \%$ \\
Deoxysodium cholate & $10 \pm 0.3$ & $1.5 \%$ \\
Ephedrine hydrochloride & $650 \pm 30$ & $99 \%$ \\
TAT-HaFGF & $638 \pm 30$ & $97 \%$ \\
\hline
\end{tabular}

$n=4$. tory glomerular layer of the olfactory bulb were counted and compared in all intranasal treatment groups using a one-way ANOVA. No significant difference was found in the number of olfactory sensory neurons between all intranasal treatment groups (Figs. 2b, 3b). Additionally, HE stained sections from each treatment group demonstrated excellent tissue structure and histology (Fig. 4). No obvious pathological lesions were detected in the heart, liver, spleen and lung in any of the groups of rats following administration of TAT-HaFGF formulations (images not shown), which were in accordance with the condition recorded in the saline control group.

Mucosal Absorption Characteristics of Intranasal Administration. Distribution of TAT-HaFGF in Tissues Distribution of ${ }^{125}$ I-TAT-HaFGF following intranasal administration was measured in SD rats and the levels of radioactivity in blood, nasal cavity, thymus, and brain tissues are shown in Table 3.

Nasal absorption of ${ }^{125}$ I-TAT-HaFGF into the systemic circulation was rapid, and the plasma level remained almost constant until $1 \mathrm{~h}$ post application (Fig. 5). At $15 \mathrm{~min}$ after intranasal administration of ${ }^{125}$ I-TAT-HaFGF, concentrations of radioactivity were observed in the hypophysis, olfactory bulb, brainstem and cerebellum. At $30 \mathrm{~min}$, stronger radioactive signals were observed in the brainstem, cerebrum, and cervical spinal cord (CSpC) and the signals became weak at $60 \mathrm{~min}$ in the brainstem, cerebellum, cerebrum and spinal cord.
A
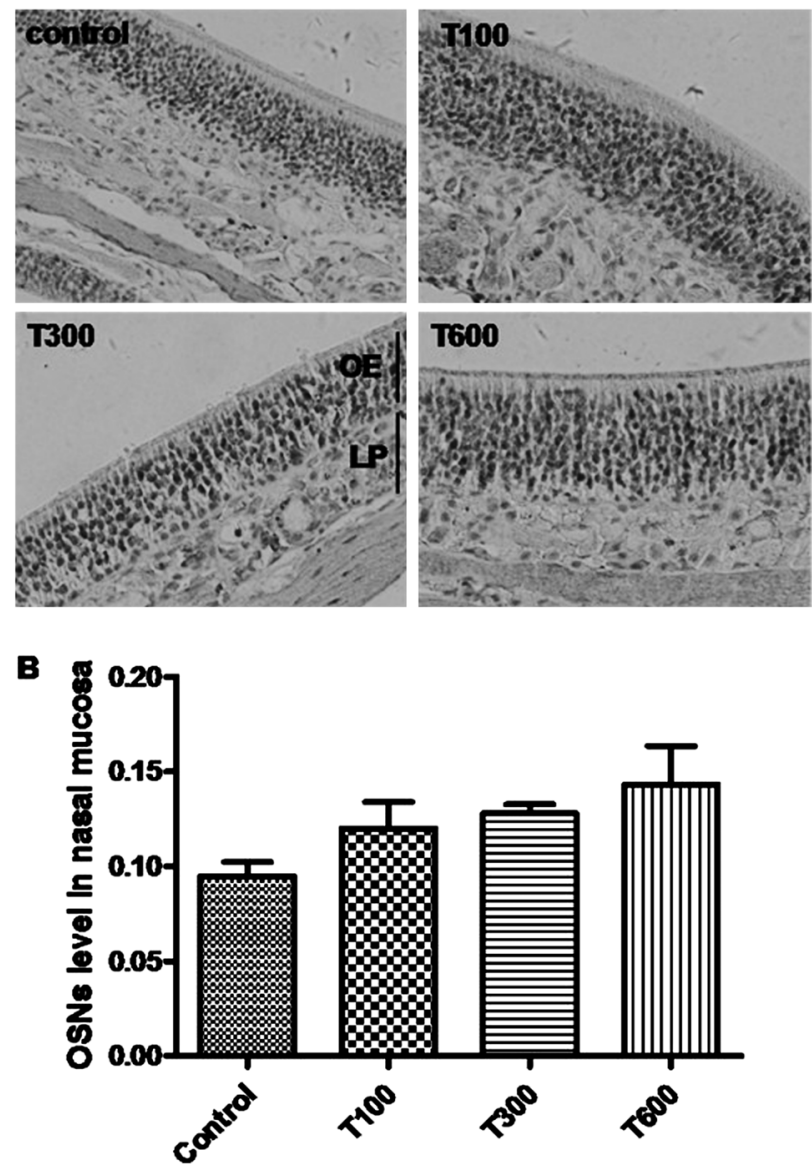

Fig. 2. The Level and Distribution of Olfactory Sensory Neurons (OSNs) in Nasal Mucosa Following Intranasal Administration of TAT-HaFGF to SD Rats for Five Weeks

(A) Morphology of the olfactory nerve in the nasal mucosa $(200 \times)$. (B) The percentage of the sum of OMP deposit areas as compared with the total area of epithelium $(\mathrm{OE})$ and lamina propria (LP) of the nasal mucosa. Five sections per group were analyzed using Image-Pro plus 6.0 software to measure the number of OMP positive plaques in the nasal mucosae. 
A
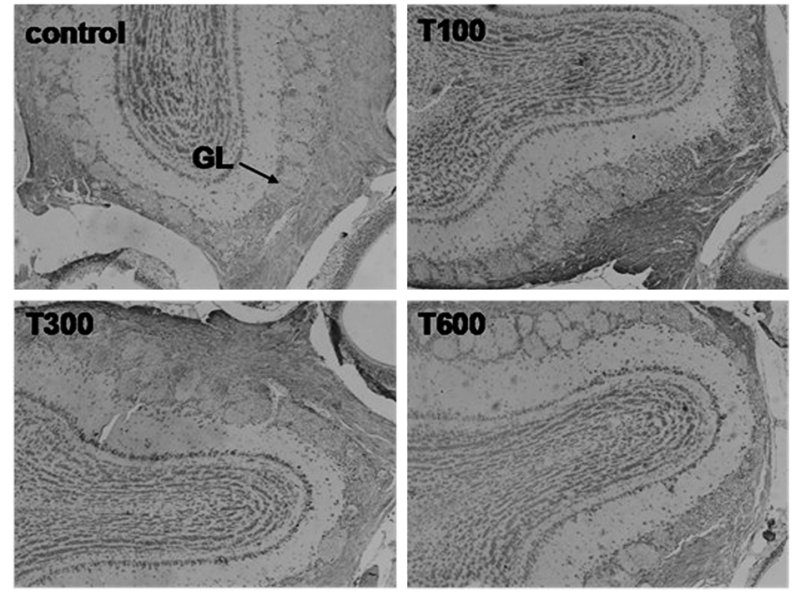

B

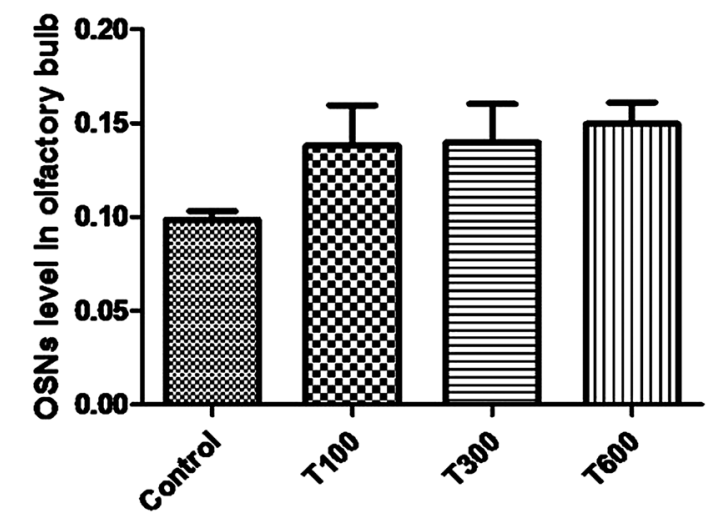

Fig. 3. The Level and Distribution of OSNs in Olfactory Bulb Following Intranasal Administration of TAT-HaFGF to SD Rats for Five Weeks

(A) Morphology of the olfactory nerve in the olfactory bulb $(100 \times)$. (B) The percentage of the sum of OMP deposit areas as compared with the total area of olfactory glomerular layer (GL) of olfactory bulb in the micrograph. $n=5$ for each group.

The intensity of ${ }^{125}$ I-TAT-HaFGF radioactivity in the whole brain, olfactory bulb, brainstem, cerebellum and cervical spinal cord reached its maximum at $30 \mathrm{~min}$ and then decreased at 60 min (Fig. 5).

Transport Visualization of TAT-HaFGF: In order to obtain direct evidence about the olfactory transfer of TAT-HaFGF, TAT-HaFGF were stained red on the nasal slice after $30 \mathrm{~min}$ following intranasal administration. DAPI, a nucleus-specific dye that emits blue fluorescence, was used to detect individual cells. Since olfactory cells are the only sensory neurons with their cell bodies in the nasal epithelium, OMP positive staining in the epithelia (shown in green in Fig. 6) were considered as olfactory epithelia.

The olfactory cells in the olfactory mucosa are bipolar sensory neurons, projecting a dendrite to the surface of the nasal epithelium and a long axon toward the olfactory bulb, which is beneficial for transport of TAT-HaFGF across the epithelium. Furthermore, red fluorescence (TAT-HaFGF) was observed in the connective tissue around the olfactory nerve bundles (Figs. 6A, B). In the nasal mucosa near the cribriform plate (Fig. 6C), only small doses of TAT-HaFGF were colocalized within nerve bundles, and more of the TAT-HaFGF were distributed around the olfactory nerve bundles. These phenomena indicated that TAT-HaFGF was transferred into the olfactory nerve bundles or their surrounding connective tissue.

\section{DISCUSSION}

Nasal cilia are mobile hair like appendages extending from the surface of the nasal epithelial cells that move in a well organized and coordinated way to propel the overlying mucus layer toward the throat. This contributes to the body's primary nonspecific defense mechanism by propelling potentially hazardous substances inside the nasal cavity. This function of the cilia emphasizes that the constituents of preparations intended for nasal delivery should not adversely affect the clearance system, especially in the chronic therapy. In the TAT-HaFGF groups, the morphology and integrity of the cilia on the toad palate, were similar to those of the normal saline group, with intact, dense cilia (Fig. 1). These results suggest that the nasal ciliotoxicity of TAT-HaFGF is negligible.

OMP immunohistochemical staining was done for longterm drug safety evaluation. OMP is expressed late in cellular development and is widely used as a marker of mature OSNs. ${ }^{14)}$ OMP immunohistochemical staining demonstrated that intranasal TAT-HaFGF does not cause damage to tissue structure or the number of OSNs (Figs. 2-4). HaFGF, a neurotrophin-like growth factor in the brain, plays important roles in the development, differentiation and regeneration of brain neurons. ${ }^{27)}$ Olfaction disorder almost is the accompaniment of $\mathrm{ND},{ }^{28,29)}$ this result may associated with the damage and apoptosis of OSNs. Although there is no siginicant difference in OSNs level between all groups, it was likely higher that the trends in OSNs level in TAT-HaFGF-treated groups than that in the control group as shown in Figs. $2 b$ and $3 b$. This result is expecting to improving the olfactory functions of ND. The two facts that HaFGF could improve the functions of cholinergic nerve $\mathrm{e}^{21)}$ and the cholinergic system modulates several as- 

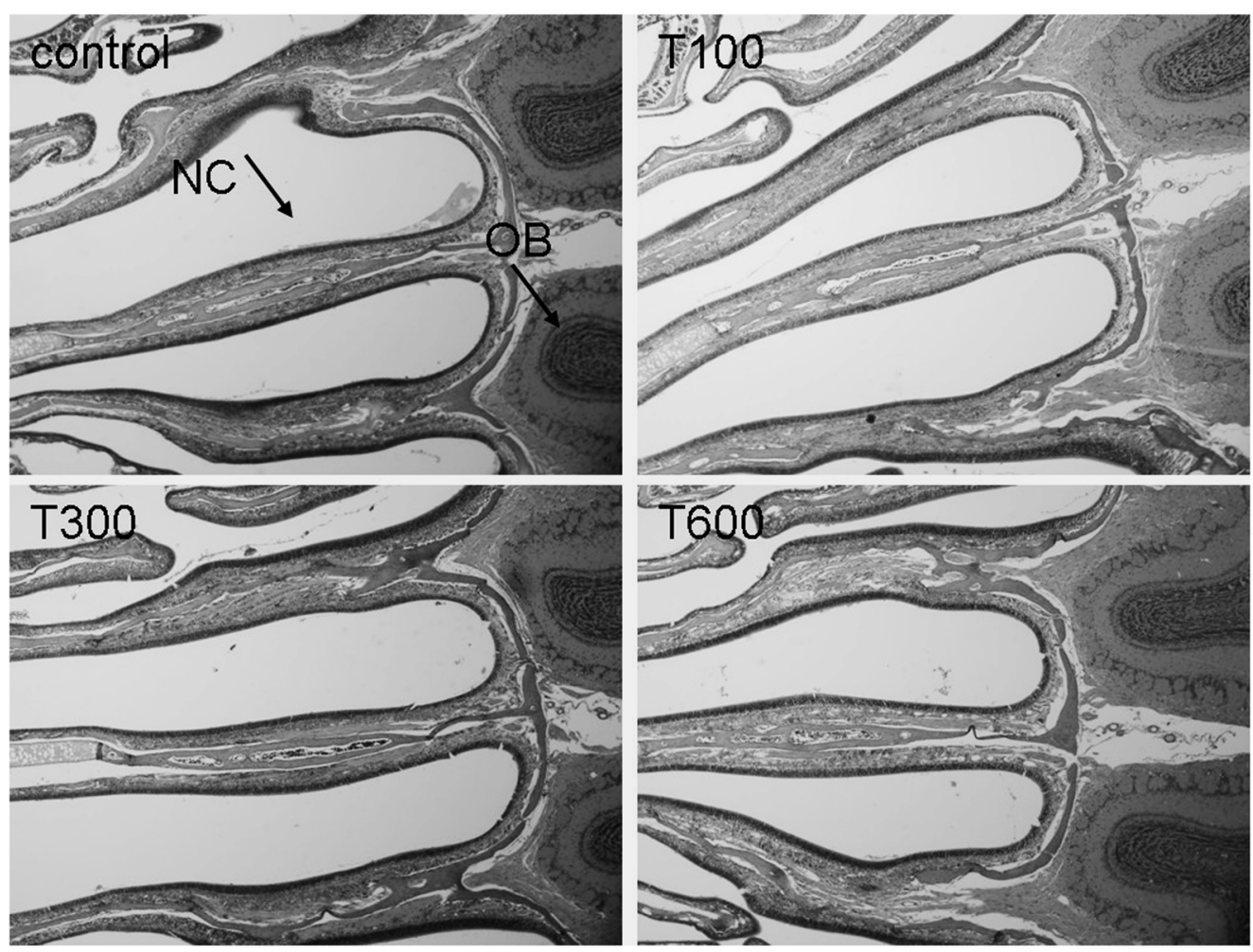

Fig. 4. SD Rats Were Treated with Intranasal Administration of TAT-HaFGF for Five Weeks

The olfactory bulbs and nasal cavity were prepared for HE staining and demonstrated excellent tissue structure and histology of nasal cavity (NC) and olfactory bulbs (OB), $n=5$.

Table 3. Amount-Time Profiles of Radioactivity in the Blood, Brain and Immune Glands

\begin{tabular}{lccc}
\hline \hline & \multicolumn{3}{c}{ Amount of ${ }^{125}$ I-TAT-HaFGF in tissue $(\mathrm{cpm} \cdot \mathrm{h} / \mathrm{g}$ tissue $/ \mathrm{mL}$ blood $)$} \\
\cline { 2 - 4 } Tissue & $15 \mathrm{~min}$ & $30 \mathrm{~min}$ & $60 \mathrm{~min}$ \\
\hline Blood & $601.2 \pm 223.1$ & $1296.6 \pm 135.8$ & $1454.2 \pm 287.7$ \\
Olfactory bulb & $3027.8 \pm 1368.1$ & $3255.0 \pm 1950.2$ & $2200.4 \pm 1304.3$ \\
Cerebrum & - & $249.2 \pm 13.8$ & - \\
Brainstem & $380.4 \pm 53.4$ & $1194.6 \pm 1270.2$ & - \\
Hypophysis & $12620.2 \pm 1337.2$ & $9526.3 \pm 195.7$ & - \\
Cerebellum & $606.0 \pm 83.8$ & $261.2 \pm 1.4$ & - \\
Cervical spinal cord & - & $327.2 \pm 80.2$ & $363.7 \pm 95.9$ \\
Thymus & $291.9 \pm 135.4$ & $35245.7 \pm 10759.0$ & $78711.1 \pm 30145.5$ \\
With thyroid's throat & $18539.4 \pm 6250.2$ & $42746.6 \pm 25905.5$ & $39984.8 \pm 27791.8$ \\
Nasal cavity & $37394.8 \pm 22985.6$ & & \\
\hline
\end{tabular}

$n=5$.

pects of central olfactory functions were well-known. The absence of olfactory behavior experiment is the main deficiency of the study. Another possibility is that, although the structure and morphology of the olfactory epithelium remained intact, the functional cellular transduction machinery within the olfactory sensory neurons, or the general olfactory processing might be damaged and cause general anosmia in the animal.

Early evidence demonstrating protein transport within olfactory sensory neurons to the olfactory bulb and other CNS areas, led to the suggestion that these nerves, or perineuronal compartments associated with them, might facilitate the initiation of some CNS diseases. ${ }^{30)}$ The present study demonstrates that TAT-HaFGF can bypass the blood-brain barrier to reach multiple sites within the brain and spinal cord likely by olfactory and trigeminal pathways.
The olfactory pathway has been reported to play a major role in the delivery of therapeutics directly to the CNS following intranasal administration. ${ }^{31)}$ The nasal barrier to the brain might be weakened because the olfactory cells regenerate every 3-4 weeks due to exposure to external toxins. ${ }^{32)}$ In addition, the continuous perineurial channels generated by the olfactory ensheathing cells that envelop the axons of olfactory cells are kept open. ${ }^{33)}$ As a result, intranasally administered TAT-HaFGF may reach the brain via extracellular transport along olfactory nerves (Fig. 6). Furthermore, neural projections of the olfactory bulb extend into multiple rostral brain tissues such as the olfactory tract, anterior olfactory nucleus, and piriform cortex ${ }^{9)}$ (Fig. 7). Therefore, the significant TATHaFGF distribution in rostral brain areas neighboring the olfactory bulb is mainly due to the olfactory pathway. 


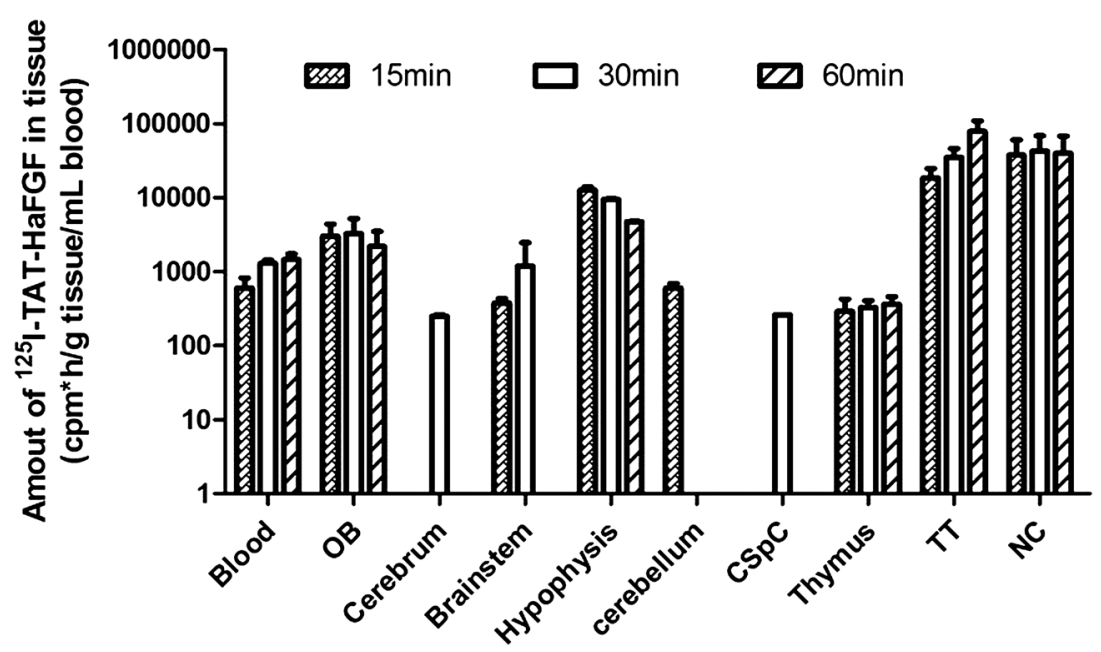

Fig. 5. Distribution of ${ }^{125} \mathrm{I}-\mathrm{TAT}-\mathrm{HaFGF}$ in Blood, Olfactory Bulb (OB), Cerebrum, Brainstem, Hypophysis, Cerebellum, Cervical Spinal Cord (CSpC), Thymus, with Thyroid's Throat (TT), and Nose Cavity (NC)
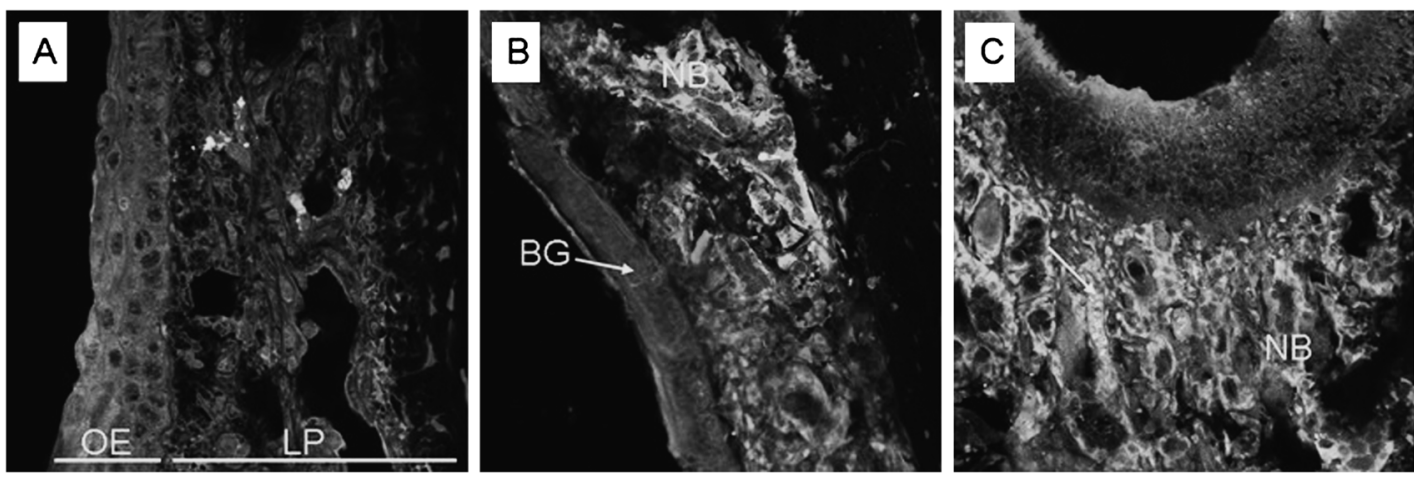

Fig. 6. Distribution of TAT-HaFGF (Red) in Slices A, B (the Nasal Mucosa near the Wing of Nose), and C (the Nasal Mucosa near the Cribriform) after 30 min Following the Intranasal Administration of TAT-HaFGF to Rats at the Dose of $300 \mu \mathrm{g} / \mathrm{kg}$, Fluorescence Was Observed in the Olfactory Epithelium (OE) and Lamina Propria (LP)

Cell nuclei were stained with DAPI (blue). Olfactory nerves were immunolabeled with OMP (green). Co-location (yellow) of the green and red fluorescence is shown by arrows. BG: Bowman gland, NB: nerve bundle $(200 \times)$. (Color images were converted into gray scale.)

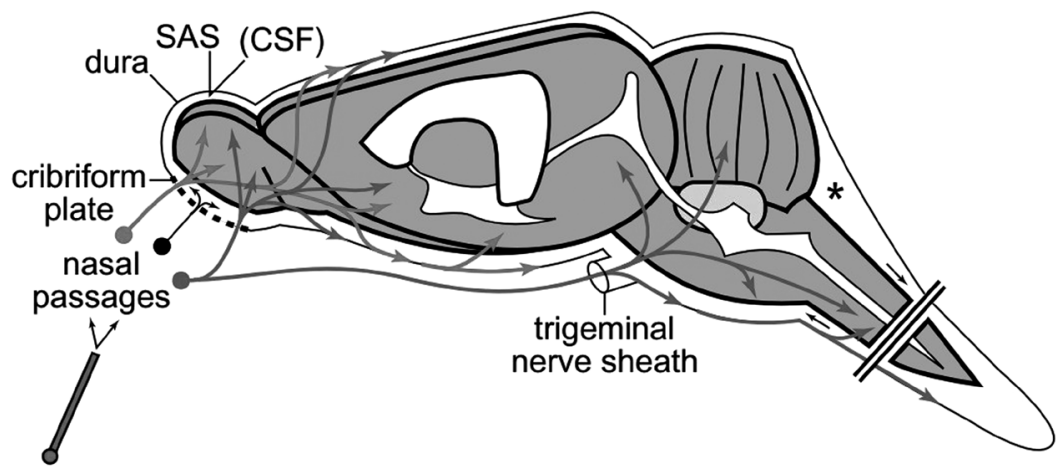

Fig. 7. Extracellular Pathways into the CNS Following Intranasal Administration

Intranasally applied proteins are rapidly transported into CNS tissue by a formerly identified pathway associated with the peripheral olfactory system (shown in red), connecting the nasal passages and olfactory bulb (OB)/rostral brain, and a newly identified pathway associated with the peripheral trigeminal system (shown in blue), connecting the nasal passages and the brainstem. Cisternal sampling in rats (asterisk) has demonstrated that some molecules, mostly smaller molecular weight solutes, can rapidly enter the CSF after intranasal administration (shown in black). (Color images were converted into gray scale.)

The trigeminal nerve enters the brainstem at the pons and some of its nerve endings terminate beneath the nasal epithelial surface, which provides a possible entrance for intranasally administrated TAT-aFGF into the brain (Fig. 7). In the present study, high concentrations of TAT-HaFGF were detected in the cervical spinal cord and cerebellum (Fig. 5), demonstrat- ing the involvement of the trigeminal nerves. Nevertheless, considering the rapid delivery shown by high radioactivity in the cervical spinal cord $15 \mathrm{~min}$ after intranasal administration (Fig. 5), an extracellular mechanism is most likely to be the predominant mode of transport into the CNS via the trigeminal nerve pathway. ${ }^{34)}$ 
Generally, neurodegenerative diseases occur in specific regions of the brain, therefore efficient drug delivery is required, not only to the brain in general, but to these specific regions. For example, Alzheimer's disease (AD), a neurodegenerative disease afflicting $c a .35$ million people worldwide in $2009,{ }^{35}$ ) has been demonstrated to damage the cerebral cortex and hippocampus; two areas near to the olfactory bulb. In addition, over the past 30 years, studies have shown early olfactory deficits in AD that spread across the olfactory spectrum with as the disease worsens. ${ }^{36,37)} \mathrm{A} \beta$ alters the activity of the OB network, resulting in a later inability to process olfactory information, as occurs in patients with $\mathrm{AD}$ and in transgenic animals. $^{38,39)}$

HaFGF plays significant roles in the development, differentiation and regeneration of brain neurons, and have similar functions to neurotrophin, such as nerve growth factors (NGF) and brain-derived neurotrophic factor (BDNF), which have been selected as new potential drugs for the treatment of AD. $^{40,41)}$ TAT-HaFGF recombined in our lab retains the neuroprotective activities of HaFGF. TAT, a protein transduction domain with a strong positive charge, could combine with the negatively charged nasal mucosa. ${ }^{14)}$ It is expected that positively charged TAT can interact electrostatically with the nasal mucosal layer, and therefore retain the entrapped TAT-HaFGF on the mucosal surface, resulting in a high local concentration for absorption. Therefore, for the treatment of AD, a new molecular designing to target the olfactory mucosa such as TATHaFGF, which has a high affinity for the olfactory epithelium, may be rational. Similarly, a drug delivery system designed to target the trigeminal nerve may be beneficial to deliver therapeutics for the treatment of the brain diseases in caudal regions, such as cerebellar atrophy. Our findings may provide valuable information for the rational design of peptides to improve the treatment of neurological and psychiatric diseases.

Acknowledgments This work was supported by the foundations from the National Science and Technology Major Project of China (No. 2009ZX09103-749), the Natural Science Foundation of China (No. 81373313), the Science and Technology Major Project of Guangdong Province (No. 2011A080502014, 2012A080201010).

\section{REFERENCES}

1) Vaka SR, Sammeta SM, Day LB, Murthy SN. Delivery of nerve growth factor to brain via intranasal administration and enhancement of brain uptake. J. Pharm. Sci., 98, 3640-3646 (2009).

2) Lv Q, Fan X, Xu G, Liu Q, Tian L, Cai X, Sun W, Wang X, Cai Q, Bao Y, Zhou L, Zhang Y, Ge L, Guo R, Liu X. Intranasal delivery of nerve growth factor attenuates aquaporins-4-induced edema following traumatic brain injury in rats. Brain Res., 1493, 80-89 (2013).

3) Prediger RD, Aguiar AS Jr, Matheus FC, Walz R, Antoury L, Raisman-Vozari R, Doty RL. Intranasal administration of neurotoxicants in animals: support for the olfactory vector hypothesis of Parkinson's disease. Neurotox. Res., 21, 90-116 (2012).

4) Hanson LR, Frey WH 2nd. Intranasal delivery bypasses the bloodbrain barrier to target therapeutic agents to the central nervous system and treat neurodegenerative disease. BMC Neurosci., 9 (Suppl. 3), S5 (2008).

5) Craft S, Baker LD, Montine TJ, Minoshima S, Watson GS, Claxton A, Arbuckle M, Callaghan M, Tsai E, Plymate SR, Green PS,
Leverenz J, Cross D, Gerton B. Intranasal insulin therapy for Alzheimer's disease and amnestic mild cognitive impairment: a pilot clinical trial. Arch. Neurol., 69, 29-38 (2012).

6) Chen XQ, Fawcett JR, Rahman YE, Ala TA, Frey IW II. Delivery of nerve growth factor to the brain via the olfactory pathway. Journal of Alzheimer's disease: JAD, 1, 35-44 (1998).

7) Dhuria SV, Hanson LR, Frey WH 2nd. Intranasal drug targeting of hypocretin-1 (orexin-A) to the central nervous system. J. Pharm. Sci., 98, 2501-2515 (2009).

8) Nonaka N, Farr SA, Kageyama H, Shioda S, Banks WA. Delivery of galanin-like peptide to the brain: targeting with intranasal delivery and cyclodextrins. J. Pharmacol. Exp. Ther., 325, 513-519 (2008).

9) Thorne RG, Pronk GJ, Padmanabhan V, Frey WH 2nd. Delivery of insulin-like growth factor-I to the rat brain and spinal cord along olfactory and trigeminal pathways following intranasal administration. Neuroscience, 127, 481-496 (2004).

10) Francis GJ, Martinez JA, Liu WQ, Xu K, Ayer A, Fine J, Tuor UI, Glazner G, Hanson LR, Frey WH 2nd, Toth C. Intranasal insulin prevents cognitive decline, cerebral atrophy and white matter changes in murine type I diabetic encephalopathy. Brain, 131, 3311-3334 (2008).

11) Ross TM, Martinez PM, Renner JC, Thorne RG, Hanson LR, Frey WH 2nd. Intranasal administration of interferon beta bypasses the blood-brain barrier to target the central nervous system and cervical lymph nodes: a non-invasive treatment strategy for multiple sclerosis. J. Neuroimmunol., 151, 66-77 (2004).

12) Ma YP, Ma MM, Ge S, Guo RB, Zhang HJ, Frey WH 2nd, Xu GL, Liu XF. Intranasally delivered TGF-betal enters brain and regulates gene expressions of its receptors in rats. Brain Res. Bull., 74, 271-277 (2007).

13) Beenken A, Mohammadi M. The FGF family: biology, pathophysiology and therapy. Nat. Rev. Drug Discov., 8, 235-253 (2009).

14) Kass MD, Moberly AH, McGann JP. Spatiotemporal alterations in primary odorant representations in olfactory marker protein knockout mice. PLoS ONE, 8, e61431 (2013).

15) Xia H, Mao Q, Davidson BL. The HIV Tat protein transduction domain improves the biodistribution of beta-glucuronidase expressed from recombinant viral vectors. Nat. Biotechnol., 19, 640-644 (2001).

16) Cecchelli R, Berezowski V, Lundquist S, Culot M, Renftel M, Dehouck MP, Fenart L. Modelling of the blood-brain barrier in drug discovery and development. Nat. Rev. Drug Discov., 6, 650-661 (2007).

17) Cooper I, Sasson K, Teichberg VI, Schnaider-Beeri M, Fridkin M, Shechter Y. Peptide derived from HIV-1 TAT protein destabilizes a monolayer of endothelial cells in an in vitro model of the bloodbrain barrier and allows permeation of high molecular weight proteins. J. Biol. Chem., 287, 44676-44683 (2012).

18) Santra S, Yang H, Stanley JT, Holloway PH, Moudgil BM, Walter G, Mericle RA. Rapid and effective labeling of brain tissue using TAT-conjugated CdS:Mn/ZnS quantum dots. Chem. Commun. (Camb.), 2005, 3144-3146 (2005).

19) Wang Y, Lin H, Lin S, Qu J, Xiao J, Huang Y, Xiao Y, Fu X, Yang Y, Li X. Cell-penetrating peptide TAT-mediated delivery of acidic FGF to retina and protection against ischemia-reperfusion injury in rats. J. Cell. Mol. Med., 14, 1998-2005 (2010).

20) Ionescu IA, Dine J, Yen Y-C, Buell DR, Herrmann L, Holsboer F, Eder M, Landgraf R, Schmidt U. Intranasally administered neuropeptide S (NPS) exerts anxiolytic effects following internalization into NPS receptor-expressing neurons. Neuropsychopharmacology, 37, 1323-1337 (2012).

21) Lou G, Zhang Q, Xiao F, Xiang Q, Su Z, Zhang L, Yang P, Yang Y, Zheng Q, Huang Y. Intranasal administration of TAT-haFGF ${ }_{14-154}$ attenuates disease progression in a mouse model of Alzheimer's disease. Neuroscience, 223, 225-237 (2012). 
22) Huang Y, Rao Y, Feng C, Li Y, Wu X, Su Z, Xiao J, Xiao Y, Feng W, Li X. High-level expression and purification of TathaFGF19-154. Appl. Microbiol. Biotechnol., 77, 1015-1022 (2008).

23) Gao X, Tao W, Lu W, Zhang Q, Zhang Y, Jiang X, Fu S. Lectinconjugated PEG-PLA nanoparticles: preparation and brain delivery after intranasal administration. Biomaterials, 27, 3482-3490 (2006).

24) Capsoni S, Giannotta S, Cattaneo A. Nerve growth factor and galantamine ameliorate early signs of neurodegeneration in antinerve growth factor mice. Proc. Natl. Acad. Sci. U.S.A., 99, 12432 12437 (2002)

25) Marks DR, Tucker K, Cavallin MA, Mast TG, Fadool DA. Awake intranasal insulin delivery modifies protein complexes and alters memory, anxiety, and olfactory behaviors. J. Neurosci., 29, 67346751 (2009).

26) Tang YM, Yang SF, Zhu B, Cui JS. Therapeutic effects of recombinant mutant human tumor necrosis factor-related apoptosis-inducing ligand on non-small lung cell cancer: an experimental with rats. Zhonghua Yi Xue Za Zhi, 85, 2021-2025 (2005).

27) Lin YL, Kuo HS, Lo MJ, Tsai MJ, Lee MJ, Huang WC, Kuo WC, Shih YH, Cheng H, Huang MC. Treatment with nerve grafts and aFGF attenuates allodynia caused by cervical root transection injuries. Restor. Neurol. Neurosci., 29, 265-274 (2011).

28) Bohnen NI, Muller ML. In vivo neurochemical imaging of olfactory dysfunction in Parkinson's disease. J. Neural Transm., 120, 571-576 (2013).

29) Djordjevic J, Jones-Gotman M, De Sousa K, Chertkow H. Olfaction in patients with mild cognitive impairment and Alzheimer's disease. Neurobiol. Aging, 29, 693-706 (2008).

30) Aebischer P, Ridet J. Recombinant proteins for neurodegenerative diseases: the delivery issue. Trends Neurosci., 24, 533-540 (2001).

31) Renner DB, Frey WH 2nd, Hanson LR. Intranasal delivery of siRNA to the olfactory bulbs of mice via the olfactory nerve pathway. Neurosci. Lett., 513, 193-197 (2012).

32) John JA, Key B. Axon mis-targeting in the olfactory bulb during re- generation of olfactory neuroepithelium. Chem. Senses, 28, 773-779 (2003).

33) Williams SK, Franklin RJ, Barnett SC. Response of olfactory ensheathing cells to the degeneration and regeneration of the peripheral olfactory system and the involvement of the neuregulins. $J$. Comp. Neurol., 470, 50-62 (2004).

34) Karceski S. Trigeminal nerve stimulator: a new treatment for seizures? Neurology, 80, e95-e97 (2013).

35) Cushman M, Johnson BS, King OD, Gitler AD, Shorter J. Prionlike disorders: blurring the divide between transmissibility and infectivity. J. Cell Sci., 123, 1191-1201 (2010).

36) Naudin M, Mondon K, Atanasova B. Alzheimer's disease and olfaction. Geriatrie et Psychologie Neuropsychiatrie du Vieillissement, 11, 287-293 (2013)

37) Seligman SC, Kamath V, Giovannetti T, Arnold SE, Moberg PJ. Olfaction and apathy in Alzheimer's disease, mild cognitive impairment, and healthy older adults. Aging Ment. Health, 17, 564-570 (2013).

38) Wesson DW, Levy E, Nixon RA, Wilson DA. Olfactory dysfunction correlates with amyloid-beta burden in an Alzheimer's disease mouse model. The Journal of Neuroscience: the Official Journal of the Society for Neuroscience, 30, 505-514 (2010).

39) Cao L, Schrank BR, Rodriguez S, Benz EG, Moulia TW, Rickenbacher GT, Gomez AC, Levites Y, Edwards SR, Golde TE, Hyman BT, Barnea G, Albers MW. Abeta alters the connectivity of olfactory neurons in the absence of amyloid plaques in vivo. Nat. Commun., 3, 1009 (2012).

40) Zhang L, Fang Y, Zeng Z, Lian Y, Wei J, Zhu H, Jia Y, Zhao X, $\mathrm{Xu}$ Y. BDNF gene polymorphisms are associated with Alzheimer's disease-related depression and antidepressant response. Journal of Alzheimer's Disease: JAD, 26, 523-530 (2011).

41) Mandel RJ. CERE-110, an adeno-associated virus-based gene delivery vector expressing human nerve growth factor for the treatment of Alzheimer's disease. Curr. Opin. Mol. Ther., 12, 240-247 (2010). 